\title{
Indirect Effects of Body Mass Index Growth on Glucose Dysregulation via Inflammation: Causal Moderated Mediation Analysis
}

\author{
Ted Chun Tat Fong \\ Center for Behavioral Health, The University of Hong Kong, Hong Kong, Hong Kong
}

\begin{abstract}
Keywords
C-reactive protein $\cdot$ Childhood obesity $\cdot$ Inflammation $\cdot$ Moderated mediation · Prediabetes . Weight gain
\end{abstract}

\begin{abstract}
Objective: No existing studies have examined the mediating role of chronic inflammation between obesity and dysregulated glucose homeostasis in adolescent samples. This study evaluated whether C-reactive protein (CRP), an inflammation biomarker, mediated the effects of growth (annual increase) in body mass index (BMI) on glycated hemoglobin $\left(\mathrm{HbA}_{1 \mathrm{c}}\right.$ ). Methods: $\mathrm{BMI}$ and biomarker data were used from wave I to wave IV of the National Longitudinal Study of Adolescent to Adult Health (Add Health study; 4,545 adolescents; mean age $=14.9$ years; $55.7 \%$ female) with valid CRP data. A causal moderated mediation analysis evaluated the direct and indirect effects of $\mathrm{BMI}$ slope on $\mathrm{HbA}_{1 \mathrm{c}}$ via CRP across gender, with demographic and clinical characteristics as model covariates. Results: The participants displayed a linear BMI growth of $0.53-0.58 \mathrm{~kg} / \mathrm{m}^{2} /$ year throughout adolescence, with substantial interindividual variation. The $\mathrm{BMI}$ slope showed positive direct and indirect effects on $\mathrm{HbA}_{1 \mathrm{c}}$ via CRP across gender, and there was a significant exposure-mediator interaction effect. A standardized increase in the $\mathrm{BMI}$ slope raised the probability of an abnormal $\mathrm{HbA}_{1 \mathrm{c}}$ value by $6.0-8.5 \%$ in participants with various profiles. The total natural indirect effect accounted for $13.3-15.9 \%$ of the total effect in males and $21.2-22.7 \%$ in females. Conclusions: The findings provide support for the inflammation mechanism in the effects of adiposity on glucose homeostasis. In adolescents, excess BMI growth was linked with a higher risk of glucose dysregulation either directly or indirectly via chronic inflammation.


Fong: Indirect Effects of BMI Growth on $\mathrm{HbA}_{1 \mathrm{c}}$ via CRP in Adolescents

\section{Introduction}

The past decades have witnessed a dramatic increase in the prevalence of adolescent obesity as a worldwide epidemic [1]. Due the expected increasing trend in body mass index (BMI) during their transition to adulthood, the adolescents are prone to excess weight gain during the transition [2]. Obesity increases the risk of various metabolic diseases such as hypertension and diabetes [3]. Glycated hemoglobin $\left(\mathrm{HbA}_{1 \mathrm{c}}\right)$ is a standard diagnostic tool for diabetes and glycemic management in at-risk individuals [4], and existing studies have linked the BMI with diabetes risk among adults [5, 6] and adolescents [7, 8]. C-reactive protein (CRP) is an inflammation biomarker following the secretion of interleukin-6 [9]. Chronic inflammation has been associated with various risk factors such as lower socioeconomic status, hypertension [10], and obesity [11-13].

Through the inflammation mechanism [14], obesity induces a chronic, low-grade inflammatory response as a result of excess nutrients in metabolic cells [15]. This unresolved response could increase levels of cytokines and adipokines such as CRP within adipose tissue [16], thereby leading to insulin resistance and metabolic dysfunction [17, 18]. Systemic inflammation and elevated CRP levels were found to positively predict $\mathrm{HbA}_{1 \mathrm{c}}$ and development of diabetes in adults [19-21]. The inflammation mechanism has, however, not been well established in adolescents, where the duration of exposure to excess adiposity is relatively shorter than in adults. This could impact the induction of proinflammatory cytokines that regulates CRP secretion from the liver and alter the state of the chronic inflammatory process. A previous study [22] found no significant association between CRP and insulin resistance in youths aged 10-16 years after adjusting for obesity.

The National Longitudinal Study of Adolescent to Adult Health (Add Health study) is an ongoing population-based survey [23] that examines the health trajectories of a nationally representative sample of American adolescents into their adulthood. Recent findings of the study [24] have shown that BMI trajectories across adolescence predicted risks of inflammation and diabetes at young adulthood. No empirical studies have, however, evaluated the inflammation mechanism in the effects of adiposity on glucose dysregulation among adolescents. More in-depth research is needed to understand the complex interplay between adiposity and inflammation in the pathogenesis of glucose dysregulation. The present study aimed to build on existing findings to systematically evaluate the potential mediating role of inflammation in this sample. A better understanding of the causal pathways would elucidate the role that inflammation plays in the temporal relationship between BMI growth and glucose dysregulation in adolescents.

First, we aimed to investigate the possible indirect causal effect of growth (annual increase) in BMI on $\mathrm{HbA}_{1 \mathrm{c}}$ via CRP using causal mediation analysis [25], a novel analytic method. Since female adolescents typically display fluctuations in inflammatory markers throughout their menstrual cycles [26,27], we conducted causal mediation analysis stratified by gender. Second, there is a potential nonlinear relationship between changes in BMI and biomarkers, where the same magnitude of increase in BMI from 20 to 25 could have a different effect than a change from 25 to 30 . We tested for potential interaction effects between baseline BMI, BMI growth, and inflammation in moderated mediation models. Figure 1 shows the conceptual model where the BMI growth is hypothesized to predict higher levels of $\mathrm{HbA}_{1 \mathrm{c}}$ via CRP and both baseline BMI and BMI growth were potential moderators. 


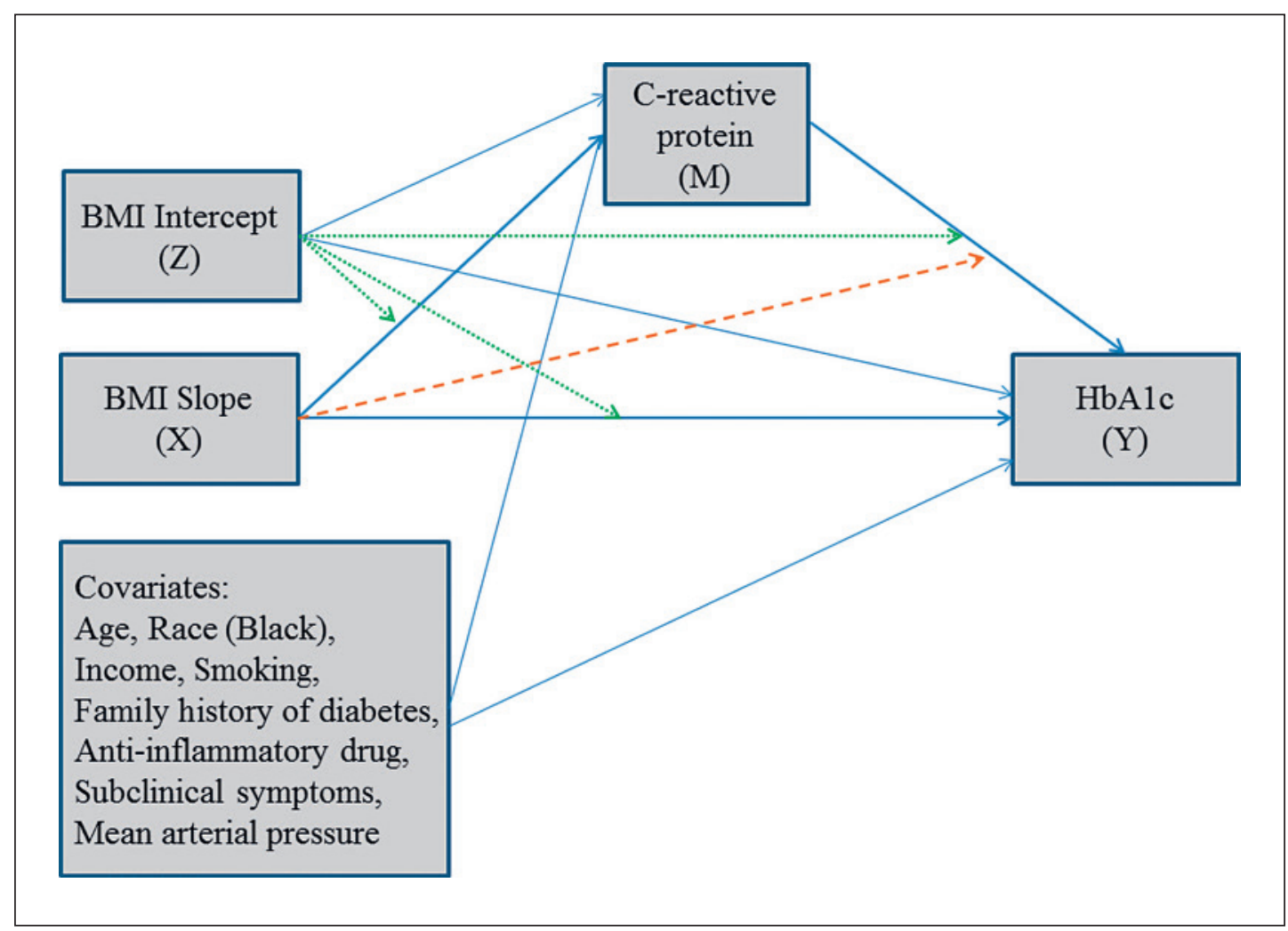

Fig. 1. Moderated mediation model of the effects of the body mass index (BMI) slope on glycated hemoglobin $\left(\mathrm{HbA}_{1 \mathrm{c}}\right)$ via $\mathrm{C}$-reactive protein. The green dotted paths denote the potential moderating effects of the BMI intercept on the relationships between the main study variables. The orange dashed path represents the potential exposure-mediator interaction effect.

\section{Subjects and Methods}

\section{Study Sample}

The study sample originates from the Add Health study. This study was a longitudinal survey of a nationally representative sample of adolescents in the USA from 1994 to 2008. It aimed to investigate the health trajectories of American adolescents into their adulthood. A sample of 132 schools was chosen in the USA under systematic sampling methods and implicit stratification with respect to region, school size, school type, and ethnicity. Details on the sampling design are available in a study report [23]. All participants provided voluntary written informed consent for participation in all aspects of the Add Health study in accordance with the University of North Carolina School of Public Health Institutional Review Board guidelines.

The present study uses the public-use data set of 5,114 participants recruited in their grades 7-12 during 1994-1995 (time 1). Three follow-up assessments were conducted in 1996 (time 2), 2001-2002 (time 3), and 2008-2009 (time 4). Participants were excluded from the present study if they had missing CRP data at time 4, were diagnosed with diabetes, or were taking diabetes medication. A total of 569 individuals were excluded from the present study, resulting in a final analytic sample of 4,545 participants. The excluded participants showed significantly lower BMI across the four time points $(d=0.17-0.29, p<0.01)$ than the included sample. The two samples did not differ significantly in age, mean arterial pressure (MAP), income, and $\operatorname{HbA}_{1 \mathrm{c}}(d<0.07, p=0.38-0.59)$. 
Fong: Indirect Effects of BMI Growth on $\mathrm{HbA}_{1 \mathrm{c}}$ via $\mathrm{CRP}$ in Adolescents

\section{Biomarker Measures}

In the Add Health study, biological specimens of metabolic and inflammatory biomarkers were collected from the sample of young adults at time 4 . $\mathrm{HbA}_{1 \mathrm{c}}$ is a measure of the average plasma glucose level over the preceding 3 months [28]. Measurement of $\mathrm{HbA}_{1 \mathrm{c}}$ does not require fasting and is more reliable than measurement of fasting plasma glucose. Trained field interviewers collected capillary whole blood samples via finger prick from the participants. The capillary whole blood spot collection cards were packaged and shipped to the Department of Laboratory Medicine at the University of Washington. $\mathrm{HbA}_{1 \mathrm{c}}$ was assayed in dried capillary whole blood spots using dried blood spot calibrators at the laboratory according to the standards of the National Glycohemoglobin Standardization Program (NGSP). The present study used the natural log-transformed $\mathrm{HbA}_{1 \mathrm{c}}$ as the primary outcome variable. To facilitate judging the clinical relevance, the continuous outcome was dichotomized into a healthy class $\left(\mathrm{HbA}_{1 \mathrm{c}}<5.7 \mathrm{mg} / \mathrm{dL}\right)$ and an abnormal class $\left(\mathrm{HbA}_{1 \mathrm{c}} \geq 5.7 \mathrm{mg} / \mathrm{dL}\right)$ using the standard criterion [3].

CRP is a biomarker synthesized by the liver that reflects the degree of inflammation [9]. CRP was assayed as an inflammatory biomarker using an enzyme-linked immunosorbent assay kit and dried blood spot calibrators under a standard protocol [29] from the capillary whole blood samples. The present study used the natural log-transformed CRP, which closely followed a normal distribution (skewness $=0.82$, kurtosis $=0.15$ ), as the mediator variable. Normally, a CRP $>3.0 \mathrm{mg} / \mathrm{dL}$ is considered a high risk [6], and extreme levels of CRP ( $>10 \mathrm{mg}$ / $\mathrm{dL}$ ) are indicative of acute viral infection. Since extreme CRP levels may not actually represent chronic inflammation, a sensitivity analysis was performed to repeat the current data analysis while removing the 614 individuals with extreme CRP values.

\section{Anthropometric Measures}

Participants' height and weight were recorded using a steel tape measure and a digital scale, respectively, across the four time points. The BMI was calculated as the ratio of weight $(\mathrm{kg})$ to the square of height $\left(\mathrm{m}^{2}\right)$. The present study focused on the intraindividual change of adiposity during adolescence and posited the BMI slope, i.e., the annual rate of BMI increase, as the exposure variable. The baseline BMI (BMI intercept) was proposed as a potential moderator of the effects of the BMI slope on the biomarkers. Given that the raw BMI varies with both age and gender during adolescence, the conventional practice is to analyze the standardized BMI $z$-scores or percentiles. Such a standardization practice is, however, not recommended for latent growth modeling, since it would alter the relationships of the variables [30]. We derived the BMI intercept and slope via multigroup latent growth models based on the raw BMI values to retain the original metric. To account for sample heterogeneity across age and gender, the latent growth models were conducted separately across gender while adjusting for the baseline age of the adolescents.

\section{Covariate Assessment}

To investigate the associations between $\mathrm{BMI}, \mathrm{CRP}$, and $\mathrm{HbA}_{1 \mathrm{c}}$, the present study controlled for demographic characteristics such as age, race (Black vs. non-Black), annual household income (in thousand USD), and smoking as covariates in the model. At time 4, blood pressure monitors were used to measure the systolic and diastolic blood pressures to derive the MAP. Other clinical variables such as family history of diabetes, count of subclinical infectious symptoms, and anti-inflammation drug use were included in the model as potential confounders. The respondents were classified as having taken anti-inflammatory drugs if they had taken any of the following medications in the past 4 weeks: nonsteroidal anti-inflammatory drugs, salicylates, Cox-2 inhibitors, inhaled corticosteroids, glucocorticoids, antirheumatics, or immunosuppressive agents. 
Fong: Indirect Effects of BMI Growth on $\mathrm{HbA}_{1 \mathrm{c}}$ via CRP in Adolescents

Table 1. Demographic and health profiles of the Add Health study participants across gender

\begin{tabular}{|c|c|c|c|c|c|}
\hline Variables & $\begin{array}{l}\text { Male } \\
(n=2,014)^{\mathrm{a}}\end{array}$ & $\begin{array}{l}\text { Female } \\
(n=2,531)^{\mathrm{a}}\end{array}$ & $\chi^{2}$ & $p$ & $d$ \\
\hline Race (Black) & $417(21)$ & $629(25)$ & 10.6 & $<0.01^{*}$ & 0.10 \\
\hline Smokes cigarettes & $1,129(56)$ & $1,405(56)$ & 0.33 & 0.56 & 0.02 \\
\hline Family history of diabetes & $152(9)$ & $191(9)$ & 0.03 & 0.87 & 0.01 \\
\hline Anti-inflammatory drug use & $524(26)$ & $843(33)$ & 28.3 & $<0.01^{*}$ & 0.16 \\
\hline Abnormal CRP (>3 mg/dL) & $596(30)$ & $1,275(50)$ & 200.0 & $<0.01^{*}$ & 0.43 \\
\hline Abnormal $\mathrm{HbA}_{1 \mathrm{c}}(\geq 5.7 \%)$ & $758(38)$ & $733(29)$ & 40.6 & $<0.01^{*}$ & 0.19 \\
\hline Variables & $\begin{array}{l}\text { Male } \\
(n=2,014)^{\mathrm{b}}\end{array}$ & $\begin{array}{l}\text { Female } \\
(n=2,531)^{\mathrm{b}}\end{array}$ & $t$ & $p$ & $d$ \\
\hline T1 age, years & $15.0(1.8)$ & $14.8(1.8)$ & 4.51 & $<0.01^{*}$ & 0.13 \\
\hline $\log$ (income) (USD) & $9.98(2.11)$ & $9.00(3.14)$ & 12.3 & $<0.01^{*}$ & 0.36 \\
\hline MAP, mm Hg & $98.0(10.3)$ & $91.2(10.4)$ & 21.7 & $<0.01^{*}$ & 0.65 \\
\hline Subclinical symptoms & $0.37(0.67)$ & $0.52(0.77)$ & -6.60 & $<0.01^{*}$ & 0.19 \\
\hline $\mathrm{T} 1 \mathrm{BMI}, \mathrm{kg} / \mathrm{m}^{2}$ & $22.8(4.4)$ & $22.5(4.6)$ & 2.63 & $<0.01^{*}$ & 0.08 \\
\hline T2 BMI, kg/m² & $23.2(4.8)$ & $23.3(5.4)$ & -0.07 & 0.94 & 0.00 \\
\hline $\mathrm{T} 3 \mathrm{BMI}, \mathrm{kg} / \mathrm{m}^{2}$ & $26.5(5.5)$ & $26.9(7.0)$ & -2.01 & 0.05 & 0.07 \\
\hline $\mathrm{T} 4 \mathrm{BMI}, \mathrm{kg} / \mathrm{m}^{2}$ & $29.1(6.6)$ & $29.6(8.2)$ & -1.83 & 0.07 & 0.05 \\
\hline $\mathrm{T} 4 \log (\mathrm{CRP}), \mathrm{mg} / \mathrm{dL}$ & $1.09(0.77)$ & $1.51(0.95)$ & -16.4 & $<0.01^{*}$ & 0.48 \\
\hline $\mathrm{T} 4 \log \left(\mathrm{HbA}_{1 \mathrm{c}}\right), \%$ & $1.89(0.09)$ & $1.87(0.09)$ & 4.81 & $<0.01^{*}$ & 0.14 \\
\hline
\end{tabular}

$\mathrm{CRP}, \mathrm{C}$-reactive protein; $\mathrm{HbA}_{1 \mathrm{c}}$, glycated hemoglobin; MAP, mean arterial pressure; BMI, body mass index. ${ }^{*} p<0.01 .{ }^{\mathrm{a}} n(\%) .{ }^{\mathrm{b}}$ Mean (SD).

\section{Data Analysis}

The demographic and health profiles of the Add Health sample were compared across gender using independent-sample $t$ tests and $\chi^{2}$ tests in SPSS 23 to explore gender differences. The BMI growth was derived via latent growth modeling using Mplus 8 [31] with the robust maximum likelihood estimator. Missing data were handled using full information maximum likelihood under the missing-at-random assumption [32]. Given the gender difference in biomarkers and adolescence stages, the present study carried out the multigroup causal mediation analysis separately in male and female participants. As shown in Figure 1, the BMI slope was the exposure variable, CRP was the mediator, and $\mathrm{HbA}_{1 \mathrm{c}}$ was the outcome. Both the BMI intercept and the BMI slope were potential moderators in the models. Model covariates included age, race, log(income), smoking, family history of diabetes, anti-inflammation medication use, count of subclinical infectious symptoms, and MAP. A series of moderated mediation models were tested to evaluate whether the interaction terms between exposure, moderator, and mediator contributed to a better model fit.

The moderated mediation models were carried out using bootstrapping with 5,000 replications. Model comparison was based on the Bayesian information criterion (BIC) and standardized root mean square residual (SRMR). A difference of $>10$ in BIC denotes substantial evidence in favor of the model with a lower BIC. The results of the causal mediation analysis [33] portrayed the total natural indirect effect (TNIE), pure natural direct effect (PNDE), and total effect of the BMI slope on $\mathrm{HbA}_{1 \mathrm{c}}$ via CRP. Supplementary analysis was performed using the logit link to predict the odds of belonging to the high-risk $\mathrm{HbA}_{1 \mathrm{c}}$ class in a probability metric [34]. The causal effects were computed with mean-centered continuous control variables (at their means) and two sets of values for the categorical control variables (race, smoking, family history of diabetes, and anti-inflammation medication use). The estimates 
Fong: Indirect Effects of BMI Growth on $\mathrm{HbA}_{1 \mathrm{c}}$ via CRP in Adolescents

Table 2. Results of the moderated mediation model with exposure-mediator interaction across gender

\begin{tabular}{|c|c|c|c|c|}
\hline & \multicolumn{2}{|l|}{ Male $(n=2,014)$} & \multicolumn{2}{|c|}{ Female $(n=2,531)$} \\
\hline & $\beta$ (SE) & $p$ & $\beta(\mathrm{SE})$ & $p$ \\
\hline \multicolumn{5}{|l|}{$\log \left(\mathrm{HbA}_{1 \mathrm{c}}\right)$ on } \\
\hline $\log (\mathrm{CRP})$ & $0.090(0.030)$ & $<0.01^{* *}$ & $0.073(0.025)$ & $<0.01^{* *}$ \\
\hline BMI slope & $0.065(0.033)$ & $0.04^{*}$ & $0.062(0.035)$ & 0.08 \\
\hline BMI slope $\times \log (\mathrm{CRP})$ & $0.121(0.050)$ & $0.02 *$ & $0.057(0.028)$ & $0.04^{*}$ \\
\hline BMI intercept & $0.110(0.032)$ & $<0.01^{* *}$ & $0.133(0.034)$ & $<0.01^{* *}$ \\
\hline Age & $0.015(0.026)$ & 0.56 & $0.017(0.022)$ & 0.45 \\
\hline Race (Black) & $0.209(0.032)$ & $<0.01^{* *}$ & $0.202(0.023)$ & $<0.01^{* *}$ \\
\hline $\log ($ income $)$ & $-0.031(0.035)$ & 0.37 & $-0.055(0.026)$ & $0.04^{*}$ \\
\hline Smoking & $0.036(0.025)$ & 0.16 & $-0.010(0.020)$ & 0.63 \\
\hline Family history of diabetes & $0.052(0.031)$ & 0.09 & $0.043(0.026)$ & 0.10 \\
\hline Anti-inflammatory drug use & $-0.004(0.024)$ & 0.87 & $-0.003(0.021)$ & 0.87 \\
\hline Subclinical symptoms & $0.014(0.025)$ & 0.57 & $0.021(0.024)$ & 0.38 \\
\hline Mean arterial pressure & $0.025(0.022)$ & 0.26 & $0.059(0.025)$ & $0.02 *$ \\
\hline \multicolumn{5}{|l|}{$\log (\mathrm{CRP})$ on } \\
\hline BMI slope & $0.331(0.029)$ & $<0.01^{* *}$ & $0.408(0.022)$ & $<0.01^{* *}$ \\
\hline BMI intercept & $0.226(0.027)$ & $<0.01^{* *}$ & $0.148(0.022)$ & $<0.01^{* *}$ \\
\hline Age & $0.023(0.024)$ & 0.33 & $0.005(0.022)$ & 0.81 \\
\hline Race (Black) & $0.004(0.017)$ & 0.82 & $0.016(0.016)$ & 0.33 \\
\hline $\log ($ income $)$ & $-0.070(0.025)$ & $<0.01^{* *}$ & $0.006(0.018)$ & 0.75 \\
\hline Smoking & $0.048(0.020)$ & $0.02^{*}$ & $0.000(0.020)$ & 0.99 \\
\hline Family history of diabetes & $0.005(0.026)$ & 0.85 & $0.007(0.022)$ & 0.75 \\
\hline Anti-inflammatory drug use & $0.062(0.024)$ & $0.02 *$ & $0.029(0.018)$ & 0.11 \\
\hline Subclinical symptoms & $0.144(0.024)$ & $<0.01^{* *}$ & $0.116(0.021)$ & $<0.01^{* *}$ \\
\hline Mean arterial pressure & $-0.016(0.027)$ & 0.56 & $-0.017(0.022)$ & 0.43 \\
\hline
\end{tabular}

were considered statistically significant if their 95\% bootstrap confidence intervals (CIs) excluded zero. The school identification code was used as a clustering variable with sample weight to account for the complex Add Health survey design.

\section{Results}

\section{Demographic and Health Profiles of the Participants}

The participants had a mean age of 14.9 years $(S D=1.8$, range $=10.4-19.9)$ at time 1 , and more than half of them were female (55.7\%) and smoked cigarettes (55.8\%). Around two-fifth $(41.2 \%)$ and one-third (33.0\%) of the sample reported abnormal CRP and $\mathrm{HbA}_{1 \mathrm{c}}$ values, respectively. As shown in Table 1, there were trivial gender differences in race, smoking rate, family history of diabetes, age, and BMI $(d<0.14)$. Female participants showed significantly more subclinical infectious symptoms and greater use of anti-inflammatory drugs $(d=0.19$ and $d=0.16$, respectively, $p<0.01)$ but lower MAP and income $(d=0.65$ and $d=0.36$, respectively, $p<0.01$ ) than males. Females were significantly more likely to have abnormal CRP and normal $\mathrm{HbA}_{1 \mathrm{c}}$ values than males. Apart from being significantly older $(d=0.35-0.39, p<0.01)$ across gender, smokers showed trivial differences in blood pressure, family history of diabetes, use of anti-inflammatory drugs, BMI, and biomarkers (CRP and $\mathrm{HbA}_{1 \mathrm{c}}$ ) from nonsmokers among both males $(d=0.01-0.13)$ and females $(d=0.01-0.09)$. 
Fong: Indirect Effects of BMI Growth on $\mathrm{HbA}_{1 c}$ via CRP in Adolescents

\section{Growth in BMI}

The mean $\left( \pm\right.$ SD) BMIs (in $\mathrm{kg} / \mathrm{m}^{2}$ ) at times $1-4$ were $22.6 \pm 4.5,23.3 \pm 5.1,26.7 \pm 6.4$, and $29.4 \pm 7.6$, respectively, indicating the expected increasing trend in BMI during adolescence. The conditional linear growth model provided an adequate fit to the data (comparative fit index $=0.99$; Tucker-Lewis index $=0.98$; root mean square error of approximation $=0.027$; SRMR $=0.016$ ). Males and females showed similar levels of baseline BMI (means $=22.7$ and 22.5 , respectively) and BMI slope (means $[\mathrm{SD}]=0.53$ [0.29] and 0.58 [0.36], respectively) with significant interindividual variation $(p<0.01)$. Older participants showed significantly higher levels of BMI intercept $(\beta=0.13-0.20, p<0.01)$ and lower levels of BMI slope $(\beta=$ -0.09 to $-0.18, p<0.01$ ) across gender. MAP significantly and positively predicted both baseline BMI ( $\beta=0.18-0.19, p<0.01)$ and BMI slope $(\beta=0.27-0.30, p<0.01)$. Females who were Black or had a family history of diabetes showed higher levels of baseline BMI $(\beta=0.13-$ $0.14, p<0.01)$ and BMI slope $(\beta=0.08-0.12, p<0.05)$.

\section{Results of the Moderated Mediation Models}

Of the specified moderated mediation models (online suppl. Table S1; for all online suppl. material, see www.karger.com/doi/10.1159/000500422), the model with exposuremediator interaction had the lowest BIC and SRMR, and addition of further interaction terms increased the BIC and SRMR. The moderated mediation model with exposure-mediator interaction was considered the optimal model in terms of model fit and parsimony. Table 2 presents the estimates of the moderated mediation model across gender. Among female participants, higher $\mathrm{HbA}_{1 \mathrm{c}}$ levels were significantly associated with being Black, having a lower income, and having a higher MAP. Among male participants, higher CRP levels were significantly linked to lower income, smoking, use of anti-inflammatory drugs, and subclinical symptoms. Controlling for the covariates, the BMI intercept significantly and positively predicted both CRP and $\mathrm{HbA}_{1 \mathrm{c}}$ across gender. The BMI slope positively predicted CRP ( $\beta=0.33-0.41, p<$ $0.01)$, which in turn positively predicted $\mathrm{HbA}_{1 \mathrm{c}}(\beta=0.07-0.09, p<0.01)$ across gender. The interaction term between BMI slope and CRP had a significant and positive effect on $\mathrm{HbA}_{1 \mathrm{c}}$ across gender $(\beta=0.06-0.13, p<0.05)$. The variance explained by the model ranged from 22.2 to $25.8 \%$ for CRP and from 11.2 to $13.3 \%$ for $\mathrm{HbA}_{1 \mathrm{c}}$ across gender.

\section{Direct and Indirect Causal Effects of BMI Growth on $H b A_{1 c}$ via CRP}

To probe the exposure-mediator interaction, online supplementary Figures 1 and 2 display the direct and indirect causal effects of BMI slope on $\mathrm{HbA}_{1 \mathrm{c}}$ across gender. In the figures, BMI slope was both the exposure and the moderator variable that varied from 2 SDs below to 2 SDs above the mean. The PNDE was not significant across gender at the BMI slope mean with the $95 \%$ CIs including zero, but it increased as a function of the BMI slope. The TNIE was statistically significant at the BMI slope mean with the $95 \%$ CIs excluding zero in females (TNIE $=0.004,95 \% \mathrm{CI}=0.002-0.007)$ and males $(\mathrm{TNIE}=0.005,95 \% \mathrm{CI}=0.002-$ $0.009)$. The TNIE increased as a function of the BMI slope and was statistically significant in the range from $1 \mathrm{SD}$ below to 2 SDs above the BMI slope mean across gender.

\section{Direct and Indirect Causal Effects of BMI Slope on Binary $H b A_{1 c}$ Class via CRP}

To judge the clinical relevance of the TNIE, causal mediation analysis was performed on the binary $\mathrm{HbA}_{1 \mathrm{c}}$ outcome. Table 3 shows the causally defined direct, indirect, and total effects of a standardized increase ( 0.29 for males and 0.36 for females) in the BMI slope from its mean on the binary $\mathrm{HbA}_{1 \mathrm{c}}$ class via CRP across gender. The TNIE, PNDE, and total effect were all statistically significant and positive across gender with their 95\% CI limits excluding zero. Among participants who were not Black, were not smoking, had no family history of diabetes, and did not use any anti-inflammatory drugs, a standardized increase in the BMI slope increased the 
Fong: Indirect Effects of BMI Growth on $\mathrm{HbA}_{1 \mathrm{c}}$ via CRP in Adolescents

Table 3. Causally defined direct, indirect, and total effects of a 1-SD increase in the BMI slope from its mean on binary $\mathrm{HbA}_{1 \mathrm{c}}$ class via $\log (\mathrm{CRP})$ across gender

\begin{tabular}{|c|c|c|c|c|}
\hline & \multicolumn{2}{|l|}{ Female } & \multicolumn{2}{|l|}{ Male } \\
\hline & estimate & $95 \% \mathrm{CI}$ & estimate & $95 \% \mathrm{CI}$ \\
\hline \multicolumn{5}{|c|}{ Non-Black, nonsmoking, no history of diabetes, not using anti-inflammatory drugs } \\
\hline Total natural IE & $0.015^{*}$ & $0.004-0.027$ & $0.008^{*}$ & $0.001-0.016$ \\
\hline Pure natural DE & $0.051^{*}$ & $0.025-0.077$ & $0.052 *$ & $0.029-0.075$ \\
\hline Total effect & $0.066^{*}$ & $0.041-0.092$ & $0.060^{*}$ & $0.035-0.084$ \\
\hline \multicolumn{5}{|c|}{ Black, smoking, history of diabetes, and using anti-inflammatory drugs } \\
\hline Total natural IE & $0.018^{*}$ & $0.004-0.030$ & $0.013^{*}$ & $0.001-0.026$ \\
\hline Pure natural DE & $0.068^{*}$ & $0.036-0.094$ & $0.068^{*}$ & $0.039-0.098$ \\
\hline Total effect & $0.085^{*}$ & $0.056-0.110$ & $0.082 *$ & $0.055-0.109$ \\
\hline
\end{tabular}

CI, confidence interval; IE, indirect effect; DE, direct effect. * $p<0.05$.

likelihood of being in the abnormal $\mathrm{HbA}_{1 \mathrm{c}}$ class by $6-6.6 \%$ across gender. The TNIE accounted for 13.3 and $22.7 \%$ of the total effect in males and females, respectively. Among participants who were Black, were smoking, had a family history of diabetes, and used anti-inflammatory drugs, a standardized increase in the BMI slope increased the probability by $8.2-8.5 \%$ across gender. The TNIE accounted for 15.9 and $21.2 \%$ of the total effect in males and females, respectively.

\section{Discussion}

The present study is the first to systematically investigate the direct and indirect effects of longitudinal changes in $\mathrm{BMI}$ on $\mathrm{HbA}_{1 \mathrm{c}}$ via $\mathrm{CRP}$ using the novel causal mediation modeling. Stratified causal mediation analysis showed consistent findings across gender with identical TNIEs in Table 3. Both baseline BMI and BMI slope showed comparable total effects on $\mathrm{HbA}_{1 \mathrm{c}}$ across gender. For adolescents with an excess growth rate in BMI $\left(0.82\right.$ and $0.94 \mathrm{~kg} / \mathrm{m}^{2} /$ year in males and females) compared to the average, the likelihood of having abnormal $\mathrm{HbA}_{1 \mathrm{c}}$ values increased significantly by $6-8 \%$. The adiposity effect showed an increment of $21 \%$ compared to the sample proportion (33.0\%) of abnormal $\mathrm{HbA}_{1 \mathrm{c}}$ values. Around one-sixth to one-fifth of this effect can be attributed to the inflammatory response of CRP. Furthermore, the results of Table 3 appear to suggest a greater health risk for the young adults who were Black, were smoking, had a family history of diabetes, and were using anti-inflammatory drugs in terms of both direct and indirect effects via CRP.

Given the lack of relevant studies on moderated mediation effects in this context, the present study adopted an exploratory approach and hypothesized a number of potential paths among the study variables. In particular, we tested for the moderating effects of BMI intercept (the green dotted paths) and the exposure-mediator interaction term (the orange dashed path). The moderated mediation analysis revealed some interesting findings. On the one hand, though adolescents with a higher baseline BMI displayed higher CRP and $\mathrm{HbA}_{1 \mathrm{c}}$ levels in young adulthood, the baseline BMI did not moderate the relationships between the BMI slope and the biomarkers. This suggests that the adiposity effect on $\mathrm{CRP}$ and $\mathrm{HbA}_{1 \mathrm{c}}$ remains consistent over baseline BMI levels across gender. On the other hand, the present study found a significant exposure-mediator interaction effect on $\mathrm{HbA}_{1 c}$, where the direct and indirect causal effects of BMI slope on $\mathrm{HbA}_{1 \mathrm{c}}$ via CRP increased as a function of the BMI slope. This result implies that adolescents with excess BMI growth are susceptible to a greater health risk of glucose dysregu- 
Fong: Indirect Effects of BMI Growth on $\mathrm{HbA}_{1 \mathrm{c}}$ via CRP in Adolescents

lation. The findings of the present study provide empirical support for the inflammatory mechanism in that CRP could possibly mediate the effects of adiposity on glucose dysregulation. Furthermore, a somewhat greater proportion of the total effect of adiposity on $\mathrm{HbA}_{1 \mathrm{c}}$ via CRP was found in females than in males. This plausibly suggests a stronger mediating role of inflammation in the adiposity effect, which could be related to the higher fat mass at a given BMI in female adolescents.

\section{Strengths and Limitations of the Study}

This study has a number of strengths. First, it utilized a nationally representative sample of adolescents recruited via systematic sampling methods. The Add Health study had high response rates (77.4-88.6\%) over a long follow-up period of 14 years across adolescence. The use of post-stratification weights in the analysis permitted generalization of the results to other adolescent samples in the USA. The second strength is the innovative use of the causal moderated mediation technique to evaluate the effects of BMI growth on $\mathrm{HbA}_{1 \mathrm{c}}$ via CRP. For the binary $\mathrm{HbA}_{1 \mathrm{c}}$ class, the conventional $\mathrm{a} \times \mathrm{b}$ product formula is not valid for mediation analysis. The current study applied the causal effect formula to decompose the total effect of adiposity on $\mathrm{HbA}_{1 \mathrm{c}}$ into causally defined direct and indirect effects. Third, this study included multiple sources of data such as biomarkers and anthropometric measures and avoided the common method bias.

Several study limitations should be noted. First, though CRP was proposed as the mediator that predicted $\mathrm{HbA}_{1 \mathrm{c}}$ based on the inflammation mechanism, both CRP and $\mathrm{HbA}_{1 \mathrm{c}}$ were concurrently assessed at time 4 . The causal effect of CRP on $\mathrm{HbA}_{1 \mathrm{c}}$ should be interpreted with caution given the possibility of a reciprocal effect of $\mathrm{HbA}_{1 \mathrm{c}}$ on CRP. The upcoming fifth wave of the Add Health study in 2018/2019 would provide valuable follow-up biomarker data to elucidate the causal relationship. Second, our moderated mediation model accounted for around one-eighth of the total variance in $\mathrm{HbA}_{1 \mathrm{c}}$. Apart from the inflammatory pathways, the association between obesity and glucose dysregulation could be explained by alternative endocrine, neural, and cell-intrinsic mechanisms [35, 36]. Future studies should explore the dynamic interplay of these mechanisms in the pathophysiology of glucose dysregulation to clarify personalized interventions for individuals with diverse profiles.

Third, $\mathrm{HbA}_{1 \mathrm{c}}$ alone may not be the perfect way to define glucose disturbance in younger populations, especially women. For instance, an adolescent with normal glucose tolerance but high glucose consumption could have a higher $\mathrm{HbA}_{1 \mathrm{c}}$ value than a person with impaired glucose tolerance consuming little carbohydrate. One should note the potential influence of glucose tolerance on the issue of glucose homeostasis. Fourth, more than one-tenth of the original Add Health sample showed abnormally high levels of CRP ( $>10 \mathrm{mg} / \mathrm{dL})$ at time 4 . This subgroup has been associated with obesity, female gender, low income, and poorer perceived health. For sample completeness and investigation of a dose-response relationship of CRP, the present study did not exclude this subgroup from the analysis, and the sensitivity analysis conducted after having removed this subgroup provided similar results. Further studies could investigate the associated factors and health risks of such significant CRP elevations in this subgroup. Fifth, the absence of randomization in the Add Health study suggests the plausibility of unmeasured confounding among the study variables. Despite the inclusion of various covariates, our analysis did not control for potential confounders such as genetics, perceived stress, and medication use. Unmeasured mediator-outcome confounding could have biased the direct and indirect causal effects.

\section{Practical Implications}

The obesity epidemic has brought massive economic costs to societies worldwide [37]. The high prevalence of inflammation and glucose dysregulation in the present study under- 
Fong: Indirect Effects of BMI Growth on $\mathrm{HbA}_{1 \mathrm{c}}$ via CRP in Adolescents

scores the clinical significance of potentially adverse metabolic outcomes for these young adults. The Add Health study sample showed an alarmingly increasing BMI trend with substantial individual variation throughout adolescence. A portion of the adolescents showed a diminished growth rate of $0.22 \mathrm{~kg} / \mathrm{m}^{2} /$ year in BMI, which corresponded to a modest rise of 3 over the 14-year period. Among these young adults, the direct effect of BMI slope on $\mathrm{HbA}_{1 \mathrm{c}}$ was not statistically significant (online suppl. Fig. 1) and its indirect effect on $\mathrm{HbA}_{1 \mathrm{c}}$ via $\mathrm{CRP}$ was smaller across gender (online suppl. Fig. 2). In contrast, other adolescents displayed an excess growth rate of $0.94 \mathrm{~kg} / \mathrm{m}^{2} /$ year in BMI, implying a massive rise of 13 over the 14 years of the Add Health study. For these young adults, the excess BMI slope posed a greater health risk on $\mathrm{HbA}_{1 \mathrm{c}}$ either directly or indirectly via CRP than for their normal counterparts. Without timely intervention, this fast-increasing trend might extend well beyond adolescence into middle adulthood and lead to irreversible damage to their metabolic functioning.

To prevent or delay the development of diabetes, preventive interventions need to be available to the high-risk young adults with excess BMI growth or chronic inflammation via the following three approaches. First, weight control programs might aim at dietary restriction to reduce the intake of fat and calories in order to control BMI growth. Second, lifestyle interventions [38] could attain behavioral changes and raise the level of physical activity of these young adults, which could in turn decrease the proportion of prediabetic patients who progress to diabetes. Third, alterations could be made to the daily diet to increase the intake of micronutrients such as phytochemicals that are anti-inflammatory in nature and abundant in fruits, vegetables, and legumes [39]. These micronutrients could disrupt inflammatory signaling and ameliorate the chronic inflammatory response of the adolescents in the long run. Effective interventions should attempt to raise public awareness of the issue of chronic inflammation and its clinical consequences [40]. Future waves of Add Health study data can provide further information on the developmental trend of diabetes and cardiovascular diseases among this young cohort of American adults as they enter middle adulthood.

\section{Acknowledgements}

This study uses data from the Add Health study, a program project funded by Grant P01-HD31921 from the Eunice Kennedy Shriver National Institute of Child Health and Human Development and directed by Kathleen Mullan Harris and designed by J. Richard Udry, Peter S. Bearman, and Kathleen Mullan Harris at the University of North Carolina at Chapel Hill. Special acknowledgment is due to Ronald R. Rindfuss and Barbara Entwisle for assistance in the original design. Information on how to obtain the Add Health data files is available on the Add Health website (http://www.cpc.unc.edu/addhealth). This publication was submitted by the first author to The University of Hong Kong for the award of the degree of Doctor of Philosophy. The author would like to express his thanks to Prof. Rainbow T.H. Ho and Prof. Paul S.F. Yip for their comments on earlier drafts of the manuscript and the two anonymous reviewers for their valuable remarks on the manuscript.

\section{Statement of Ethics}

The study protocol on this secondary data analysis has been approved by the Human Research Ethics Committee of the university (Ref. No. EA1707021) for this secondary analysis. All participants have given their written informed consent to participate in the original Add Health study. 


\section{Disclosure Statement}

The author has no conflicts of interest to declare.

\section{Funding Sources}

No direct support was received from Grant P01-HD31921 or any other grant for this analysis.

\section{References}

1 Abarca-Gómez L, Abdeen ZA, Hamid ZA, Abu-Rmeileh NM, Acosta-Cazares B, Acuin C, et al.; NCD Risk Factor Collaboration (NCD-RisC). Worldwide trends in body-mass index, underweight, overweight, and obesity from 1975 to 2016: a pooled analysis of 2,416 population-based measurement studies in 128.9 million children, adolescents, and adults. Lancet. 2017 Dec;390(10113):2627-42.

2 Lee H, Lee D, Guo G, Harris KM. Trends in body mass index in adolescence and young adulthood in the United States: 1959-2002. J Adolesc Health. 2011 Dec;49(6):601-8.

3 American Diabetes Association. Diagnosis and classification of diabetes mellitus. Diabetes Care. 2014 Jan;37 Suppl 1:S81-90.

4 International Expert Committee. International Expert Committee report on the role of the A1C assay in the diagnosis of diabetes. Diabetes Care. 2009 Jul;32(7):1327-34.

5 Wheelock KM, Fufaa GD, Nelson RG, Hanson RL, Knowler WC, Sinha M. Cardiometabolic risk profile based on body mass index in American Indian children and adolescents. Pediatr Obes. 2017 Aug;12(4):295-303.

6 Pearson TA, Mensah GA, Alexander RW, Anderson JL, Cannon RO 3rd, Criqui M, et al.; Centers for Disease Control and Prevention; American Heart Association. Markers of inflammation and cardiovascular disease: application to clinical and public health practice: a statement for healthcare professionals from the Centers for Disease Control and Prevention and the American Heart Association. Circulation. 2003 Jan;107(3):499511.

7 Boyer BP, Nelson JA, Holub SC. Childhood body mass index trajectories predicting cardiovascular risk in adolescence. J Adolesc Health. 2015 Jun;56(6):599-605.

8 Tirosh A, Shai I, Afek A, Dubnov-Raz G, Ayalon N, Gordon B, et al. Adolescent BMI trajectory and risk of diabetes versus coronary disease. N Engl J Med. 2011 Apr;364(14):1315-25.

9 Pepys MB, Hirschfield GM. C-reactive protein: a critical update. J Clin Invest. 2003 Jun;111(12):1805-12.

10 Sesso HD, Buring JE, Rifai N, Blake GJ, Gaziano JM, Ridker PM. C-reactive protein and the risk of developing hypertension. JAMA. 2003 Dec;290(22):2945-51.

11 Rode L, Nordestgaard BG, Weischer M, Bojesen SE. Increased body mass index, elevated C-reactive protein, and short telomere length. J Clin Endocrinol Metab. 2014 Sep;99(9):E1671-5.

12 Choi J, Joseph L, Pilote L. Obesity and C-reactive protein in various populations: a systematic review and metaanalysis. Obes Rev. 2013 Mar;14(3):232-44.

13 Abo-Zaid G, Morrissey K. Examining the association between C-Reactive protein and obesity by using the fractional polynomial approach; applying on NHANES dataset from 2001 to 2010. J Med Stat Inform. 2017;5(1): 2.

14 Lumeng CN, Saltiel AR. Inflammatory links between obesity and metabolic disease. J Clin Invest. 2011 Jun; 121(6):2111-7.

15 Gregor MF, Hotamisligil GS. Inflammatory mechanisms in obesity. Annu Rev Immunol. 2011;29(1):415-45.

16 Ellulu MS, Patimah I, Khaza'ai H, Rahmat A, Abed Y. Obesity and inflammation: the linking mechanism and the complications. Arch Med Sci. 2017 Jun;13(4):851-63.

17 O'Rourke RW. Molecular mechanisms of obesity and diabetes: at the intersection of weight regulation, inflammation, and glucose homeostasis. World J Surg. 2009 Oct;33(10):2007-13.

18 Donath MY, Shoelson SE. Type 2 diabetes as an inflammatory disease. Nat Rev Immunol. 2011 Feb;11(2): 98-107.

19 Sabanayagam C, Shankar A, Lim SC, Lee J, Tai ES, Wong TY. Serum C-reactive protein level and prediabetes in two Asian populations. Diabetologia. 2011 Apr;54(4):767-75.

20 Freeman DJ, Norrie J, Caslake MJ, Gaw A, Ford I, Lowe GD, et al.; West of Scotland Coronary Prevention Study. C-reactive protein is an independent predictor of risk for the development of diabetes in the West of Scotland Coronary Prevention Study. Diabetes. 2002 May;51(5):1596-600.

21 Ahmadi-Abhari S, Kaptoge S, Luben RN, Wareham NJ, Khaw KT. Longitudinal association of C-reactive protein and Haemoglobin A1c over 13 years: the European Prospective Investigation into Cancer-Norfolk study. Cardiovasc Diabetol. 2015 May;14(1):61. 
Fong: Indirect Effects of BMI Growth on $\mathrm{HbA}_{1 \mathrm{c}}$ via CRP in Adolescents

22 Moran A, Steffen LM, Jacobs DR Jr, Steinberger J, Pankow JS, Hong CP, et al. Relation of C-reactive protein to insulin resistance and cardiovascular risk factors in youth. Diabetes Care. 2005 Jul;28(7):1763-8.

23 Harris KM. The add health study: design and accomplishments. Chapel Hill: Carolina Population Center, University of North Carolina at Chapel Hill; 2013.

24 Attard SM, Herring AH, Howard AG, Gordon-Larsen P. Longitudinal trajectories of BMI and cardiovascular disease risk: the National Longitudinal Study of Adolescent Health. Obesity (Silver Spring). 2013 Nov;21(11): 2180-8.

25 Muthén BO, Asparouhov T. Causal effects in mediation modeling: an introduction with applications to latent variables. Struct Equ Modeling. 2015;22(1):12-23.

26 Blum CA, Müller B, Huber P, Kraenzlin M, Schindler C, De Geyter C, et al. Low-grade inflammation and estimates of insulin resistance during the menstrual cycle in lean and overweight women. J Clin Endocrinol Metab. 2005 Jun;90(6):3230-5.

27 Gaskins AJ, Wilchesky M, Mumford SL, Whitcomb BW, Browne RW, Wactawski-Wende J, et al. Endogenous reproductive hormones and C-reactive protein across the menstrual cycle: the BioCycle Study. Am J Epidemiol. 2012 Mar;175(5):423-31.

28 Nathan DM, Turgeon H, Regan S. Relationship between glycated haemoglobin levels and mean glucose levels over time. Diabetologia. 2007 Nov;50(11):2239-44.

29 McDade TW, Burhop J, Dohnal J. High-sensitivity enzyme immunoassay for C-reactive protein in dried blood spots. Clin Chem. 2004 Mar;50(3):652-4.

30 Seltzer MH, Frank KA, Bryk AS. The metric matters: the sensitivity of conclusions about growth in student achievement to choice of metric. Educ Eval Policy Anal. 1994;16(1):41-9.

31 Muthén LK, Muthén BO. Mplus user's guide. 8th ed. Los Angeles, CA: Muthen \& Muthen; 1998-2017.

32 Little RJ, Rubin DB. Statistical analysis with missing data. John Wiley \& Sons; 2014.

33 Muthén BO. Applications of causally defined direct and indirect effects in mediation analysis using SEM in Mplus. Manuscript submitted for publication 2011. Available from: https://www.statmodel.com/download/ causalmediation.pdf.

34 Muthén BO, Muthén LK, Asparouhov T. Regression and mediation analysis using Mplus. Los Angeles (CA): Muthén \& Muthén; 2016.

35 Hardy OT, Czech MP, Corvera S. What causes the insulin resistance underlying obesity? Curr Opin Endocrinol Diabetes Obes. 2012 Apr;19(2):81-7.

36 Qatanani M, Lazar MA. Mechanisms of obesity-associated insulin resistance: many choices on the menu. Genes Dev. 2007 Jun;21(12):1443-55.

37 Wang Y, Beydoun MA. The obesity epidemic in the United States - gender, age, socioeconomic, racial/ethnic, and geographic characteristics: a systematic review and meta-regression analysis. Epidemiol Rev. 2007; 29(1):6-28.

38 Tuso P. Prediabetes and lifestyle modification: time to prevent a preventable disease. Perm J. 2014;18(3): 88-93.

39 Zhu F, Du B, Xu B. Anti-inflammatory effects of phytochemicals from fruits, vegetables, and food legumes: a review. Crit Rev Food Sci Nutr. 2018 May;58(8):1260-70.

40 Serhan CN. Treating inflammation and infection in the 21st century: new hints from decoding resolution mediators and mechanisms. FASEB J. 2017 Apr;31(4):1273-88. 